\title{
Low and high order aberrations in the eyes of patients with levothyroxine treated hypothyroidism — comparison and analysis
}

\author{
Mateusz Grzesik (1) ${ }^{1-4}$ \\ 'University I Isabel de Castilla, Burgos, Spain \\ ${ }^{2}$ School of Advanced Education, Research and Accreditation, Castellón, Spain \\ ${ }^{3}$ Optometria Mateusz Grzesik, Bydgoszcz, Poland \\ ${ }^{4}$ Nicolaus Copernicus University in Toruń, Ludwik Rydygier, Collegium Medicum in Bydgoszcz, Bydgoszcz, Poland
}

\begin{abstract}
BACKGROUND: There is an increasing number of patients in optometric practices who suffer from hypothyroidism. Thyroid diseases have an impact on the ocular condition, refraction, and tear film stability, particularly when thyroid is hyperreactive. This research is focused on the optical condition of the eyes in patients with hypothyroidism treated with levothyroxine to assess its importance during refraction exams.

MATERIAL AND METHODS: A group of 43 patients was examined, their medical case history was taken, and aberrometry was performed. A research and control groups were selected for statistical analysis of the obtained results. Refractive condition, keratometry, whole eye aberrometry, and separately cornea and internal aberrometry were performed with the use of iTrace aberrometer, based on ray-tracing aberrometry and Placido disk corneal topography. RESULTS: Statistically significant differences with a threshold of $\mathrm{p}=0.05$ were total eye horizontal coma and corneal horizontal secondary coma. Three values were slightly above the threshold - whole eye oblique tetrafoil, corneal vertical pentafoil, and internal horizontal coma. All three parameters have a p-value equal to 0.06 .

CONCLUSION: Hypothyroidism treated with levothyroxine does not have a significant impact on the ocular surface or internal eye aberrations of low order. However, in case of total eye horizontal coma and secondary corneal horizontal coma, the differences are statistically significant.
\end{abstract}

KEY WORDS: hypothyroidism; ocular aberrations; aberrometry; levothyroxine

Ophthalmol J 2021; Vol. 6, 38-43

\section{INTRODUCTION}

Thyroid diseases can affect the eyes in many ways, from Graves-Basedow disease, through a change in refractive state in thyroid eye disease present in hyperthyroidism, to eye dryness in hypothyroidism [1]. Every change in refractive error, tear film morphology, and eye anatomy influences eye aberrations of both high and low order [2]. Hypothyroidism is a common thyroid disorder caused by a deficiency of thyroid hormones. Incidence of the disease increases with age, with a higher prevalence in women [3].

Hypothyroidism can be present in two forms: with full symptoms (clinical) or with no or low symptoms (subclinical) [1].

There are various causes of hypothyroidism. Primary causes are associated with thyroid gland dysfunction, i.e., iodine deficiency, autoimmune thyroiditis (such as Hashimoto thyroiditis or atrophic autoimmune thyroiditis). Primary hypothyroidism 
can also be congenital or induced by medications, thyroid aplasia or hypoplasia, and defective synthesis of thyroid hormones associated with thyroidectomy or cancer treatments. Central or secondary causes are associated with pituitary dysfunction, such as pituitary adenoma, craniopharyngioma, traumatic brain injury, Sheehan syndrome, sarcoidosis, syphilis, tuberculosis, or iron overload [4]. Furthermore, some drugs can cause hypothyroidism, i.e., carbimazole, methimazole, iodine, amiodarone, thalidomide, sunitinib, rifampicin [5].

Common symptoms not related to the eyes can manifest as increased body weight, sleepiness, memory disorders, bradycardia (slower heart rate), sense of coldness, especially in hands and feet [1]. Other symptoms include dry skin and hair, paresthesias, fatigue, memory deficits, myalgias, and arthralgias [3].

Diagnostics of hypothyroidism involve checking thyroid-stimulating hormone (TSH), thyroxine (T4), and triiodothyronine (T3) levels in the blood, as well as performing imaging of thyroid and pituitary glands to check whether any lesions are present in central hypothyroidism [6].

Eye aberrations are distortions (deviations) of a wavefront passing through ocular media from a reference (ideal) wavefront. Aberrations tend to be expressed in micrometers. However, they can be converted to dioptres. The main methods to express aberrations for aberrometers is root mean square (RMS) - the Zernike expansion coefficient and Zernike polynomials.

The coefficients are calculated using differences between the wavefront and the reference surface. Both RMS and Zernike polynomials are measured in micrometers [7]. When the light passes through ocular media, it is affected by imperfections of the eye optical system. This causes imperfections in the retinal image and, later, imperfections in the perceived image. These imperfections or aberrations can be classified in different ways. One of these classifications is with the use of Zernike polynomials. These are used to categorize the eye aberrations into two types: low-order aberrations (LOA) and high-order aberrations (HOA) [8].

Low-order aberrations can be considered from zero $(\mathrm{n}=0)$ to second-order $(\mathrm{n}=2)$ aberrations, i.e., piston, tilt (prism), defocus, and astigmatism. In ophthalmology and optometry, defocus and astigmatism are considered the most significant. High-order aberrations start from third-order $(\mathrm{n}=3)$ and higher. These include many different aberrations, such as coma, trefoil, secondary astigmatism, and spherical aberration [9].

The maps of aberrations (which are similar to corneal topography maps) show derogations of the wavefront from the reference wavefront surface. In the standard aberrometry map, all aberrations are combined, but they can be divided into separate map instances indicating each deviation. A number of each type of aberration can be shown $[8,10]$.

Aberrometry is based on different principles. Nowadays, the two most popular and recognizable are Hartmann-Shack-based aberrometry and ray-tracing aberrometry [11].

In addition to aberrometry, corneal topography can also be performed. When an aberrometer has the ability to perform both aberrometry of the whole eye and corneal topography (from which corneal aberrations can be calculated), it can indicate internal aberrations induced by the ocular lens by subtracting corneal from whole eye aberrations $[10,12]$.

With such tools, the influence of diseases such as hypothyroidism to the ocular surface, intraocular lens, and overall eye aberrations, such as refractive error, can be evaluated [8].

The aim of this study is to assess the influence of hypothyroidism treated with levothyroxine on the refractive state of the eye - higher and lower order aberrations that can occur as a result of possible changes in ocular media due to hypothyroidism.

Optometrists and ophthalmologists who perform eye exams need to think about various factors that can influence subjective and objective refraction measurements. Errors and low repeatability of these measurements can lead to wrong ocular or contact lens prescription, but also a bias in intraocular lens calculation and inaccurate measurements for wavefront-guided refractive surgery.

The purpose of this research is to investigate whether hypothyroidism, treated with levothyroxine, has an impact on aberrations of the eye. It is investigated whether hypothyroidism causes changes in aberrations of the eye, caused by changes in the cornea and crystalline lens, and whether these changes in aberrations reverse during treatment of hypothyroidism with levothyroxine.

\section{MATERIAL AND METHODS}

A group of 43 patients was examined. All patients gave informed consent for the research. The 
examination process included recording case history of the ocular condition (ocular diseases, trauma, surgery, ocular symptoms like dryness, pain, itching) and systemic diseases (cardiovascular diseases, thyroid diseases, hypertension, diabetes). The anamnesis was followed by the aberrometry with corneal topography performed on every patient. Each eye was measured three times (three whole eye aberrometry measurements and three topography scans). The recorded data for every measurement was averaged to minimize the eyeball movement's negative impact, such as tremors or microsaccades.

The device used to perform measurements was the iTrace aberrometer, based on the ray-tracing aberrometry principle with built-in Placido disks corneal topographer.

The aberrometer measurements were conducted with standard parameters, i.e., vertex distance of $12 \mathrm{~mm}$ and scan zone of $2.5 \mathrm{~mm}$, to eliminate the effect of random pupil size to amount of various aberrations.

The patients were divided into two groups, with and without hypothyroidism. The control group's inclusion criteria were age from 18 to 45 years old, no systemic or ocular diseases, and no hormonal problems. In the examined group, inclusion criteria were age from 18 to 45 years old, no ocular or systemic diseases except hypothyroidism treated with levothyroxine. The control group consisted of 32 participants (64 eyes examined, 192 measurements), and the examined group consisted of 11 participants (22 examined eyes, 66 measurements). The remaining patients were excluded from the research because of their age or other diseases that could negatively affect the results. The mean age of the control group was 26 years; in the examined group, it was 27 years (ages rounded). In the group with hypothyroidism, only females were present, while in the control group, both males and females participated in the research.

The measurements were taken in the same examination room in constant lighting conditions. All the measurements were taken manually, without the usage of automated capture of topography and aberrometry.

In every patient, a number of different eye parameters were measured, i.e.:

- angle kappa distance and degree;

- angle alpha distance and degree;

- autorefractometry (spherical, cylindrical, and axis);

- total Zernike aberrations of the eye to 27 polynomial;
- limbal diameter, keratometry;

- Zernike aberrations of the cornea to 27 polynomial;

- internal Zernike aberrations to 27 polynomial;

- dysfunctional lens index (DLI).

All the obtained data were subjected to statistical analysis, which checked if any significant differences in the parameters were present. To perform the analysis, the data used for calculations were rounded to the ninth decimal place. First polynomials, piston, and tilt in $\mathrm{X}$-axis and $\mathrm{Y}$-axis were neglected as they do not impact the optical resolution of the eye optics.

The statistical analysis was performed with the parametric and non-parametric tests. In order to evaluate differences in the standard level of numerical feature in two groups, Student's t-test for non-related variables or, alternatively, non-parametric Mann-Whitney U test were used.

The choice of parametric tests was dependent on compliance with its principal assumptions, i.e., compliance of the distributions of the studied variables with the normal distribution, verified by the Shapiro-Wilk W test. In addition, the level of statistical significance was $\mathrm{p}<0.05$. Performed measurements allow calculating mean values, median, minimal and maximum value, as well as standard deviation.

\section{RESULTS}

The obtained results are topographical maps of the cornea, aberrometry maps of Zernike polynomials, and Zernike polynomials' values of the optical system of the whole eye and cornea. Furthermore, the intraocular crystalline lens was calculated by subtracting corneal aberrations from whole eye aberrations. The results also include other physiological parameters of the eyes, i.e., simulated keratometry, refractive state - defocus, astigmatism and astigmatism axis, kappa and alpha angles, and limbal diameter. All the aberrometry results obtained for the whole eye, cornea, and lens are expressed in micrometers. The results with statistically different values in the control and tested group are presented in Table 1.

The differences in the values for total eye horizontal coma and corneal horizontal secondary coma are statistically significant. The mean total eye horizontal coma value in the control group is equal to -0.00363534 . In the examined group, the value is more positive and equal to +0.00774231 . Converse- 


\begin{tabular}{|c|c|c|c|c|c|c|c|c|c|c|c|c|}
\hline \multirow{2}{*}{ Variable } & \multicolumn{5}{|c|}{ Examined group $(n=22)$} & \multicolumn{5}{|c|}{ Control group $(n=64)$} & \multirow{2}{*}{ Z } & \multirow{2}{*}{$\mathbf{p}$} \\
\hline & Mean & Me & Min & Max & SD & Mean & Me & Min & Max & SD & & \\
\hline \multicolumn{13}{|l|}{ Total eye aberrations } \\
\hline Horizontal coma & 0.01 & 0.01 & -0.03 & 0.05 & 0.02 & -0.00 & -0.00 & -0.11 & 0.05 & 0.02 & 2.32 & 0.02 \\
\hline Oblique tetrafoil & -0.01 & -0.01 & -0.03 & 0.02 & 0.01 & 0.00 & -0.00 & -0.03 & 0.12 & 0.02 & -1.88 & 0.06 \\
\hline \multicolumn{13}{|l|}{ Corneal aberrations } \\
\hline $\begin{array}{l}\text { Horizontal secondary } \\
\text { coma }\end{array}$ & -0.00 & -0.00 & -0.00 & 0.00 & 0.00 & 0.00 & 0.00 & -0.00 & 0.00 & 0.00 & -2.16 & 0.03 \\
\hline Vertical pentafoil & 0.00 & 0.00 & 0.00 & 0.00 & 0.00 & 0.00 & 0.00 & -0.01 & 0.01 & 0.00 & 1.92 & 0.06 \\
\hline \multicolumn{13}{|l|}{ Internal aberrations } \\
\hline Horizontal coma & 0.01 & 0.00 & -0.04 & 0.05 & 0.02 & -0.00 & -0.00 & -0.10 & 0.06 & 0.02 & 1.91 & 0.06 \\
\hline
\end{tabular}

Me — median; Min — minimum; Max — maximum; SD — standard deviation; Z — Mann-Whitney U test result; $p$ — probability of test level

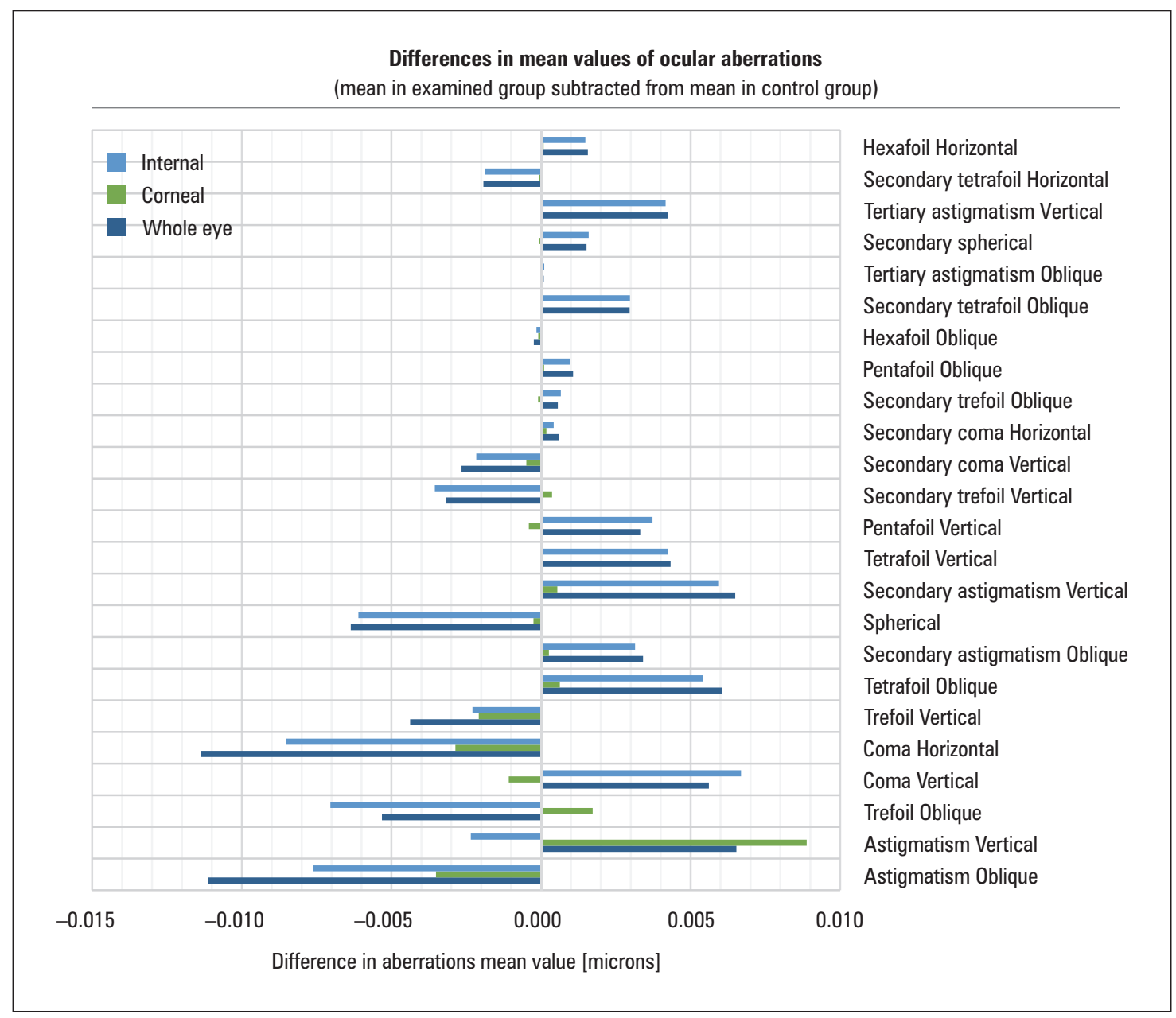

FIGURE 1. Differences in mean values of ocular aberrations (no defocus)

ly, the mean corneal horizontal secondary coma value is more positive in the control group and equal to +0.00007532 and in the examined group equal to -0.00010604 . The rest of the parameters, aberrations, refraction, keratometry, angles, and the other data are not statistically significant in the differences. The maximum and minimum values of parameters are similar in both groups.

The obtained results show that arithmetic mean values for corneal and internal (lenticular) aberra- 
tions are similar, but the whole eye aberrations are more varied (Fig. 1). Standard deviation values are similar for all aberrations (whole eye aberrations, internal aberrations, and corneal aberrations).

The $p$-values indicate that there are only two values below a threshold, which are statistically significant with $p=0.05$. Furthermore, three values are slightly above the threshold: whole eye oblique tetrafoil, corneal vertical pentafoil, internal horizontal coma. All three parameters have a p-value equal to 0.06 . Total eye horizontal coma and corneal horizontal secondary coma are below the threshold. The relatively small difference between the obtained values (whole eye oblique tetrafoil, corneal vertical pentafoil, internal horizontal coma) and the threshold could be an error due to the relatively small examined group. If more patients were enrolled in the study, the error could become negligible.

The results indicate no significant shift in spherical refraction, astigmatism, or shift in cylinder axis direction or alpha and kappa angles in control and examined group.

This means that the main astigmatism is with-the-rule and against-the-rule, with less amount of oblique astigmatism. Kappa angle is mainly directed nasally, with much more dispersion than in alpha angle, which is directed temporally but with more focus. In both groups, hypothyroidic and control, dispersion and mean direction of angles of the eye are similar.

\section{DISCUSSION}

The data from research in the field of association of eye aberrations and hypothyroidism are limited. There is one case report by Gatzioufas et al. [13]. The authors observed that corneal curvature of a patient with hypothyroidism was very high (49.7 diopters in the right and 51.0 diopters in the left eye), in comparison to the normal values recorded ten years earlier. The patient was diagnosed with hypothyroidism, with symptoms like fatigue and anorexia. In addition, his blood test indicated a thyroid-stimulating hormone (TSH) level above normal. After treating the patient with levothyroxine, the corneal curvature gradually returned to the normal values. This case report shows that untreated hypothyroidism can impact anterior eye segment morphology, and treating it with levothyroxine can lead to regression of changes caused by hypothyroidism. A larger group of patients with hypothyroidism should be examined twice - before and after starting treatment. Results should be compared to get a full view of how hypothyroidism affects the ocular surface.

A comparison of aberrations in the third group of patients with hypothyroidism before treatment could also be made. This would allow stating if any changes in corneal, internal, and total eye aberrations existed before using levothyroxine treatment and if these changes disappeared after the start of hypothyroidism treatment.

Another option is to monitor patients with hypothyroidism diagnosed early, then perform aberrometry on them and repeat measurements before and after levothyroxine treatment has been started. This would allow to monitor changes in aberrometry in the duration of treatment and show how quickly potentially existing aberrometry changes would disappear.

This research does not show if the changes are present before treatment and if levothyroxine treatment impacts the eyes. Still, it shows that the condition of eye optics in patients with treated hypothyroidism is very similar to the eye optics condition present in healthy people. This includes not only the high order aberrations but also keratometry measurements, refraction measurements, and some physiological parameters as limbal diameter (white-to-white measurement).

The obtained results and their analysis in this research show that the treated hypothyroidism does not significantly impact the cornea, lens, and overall eye condition. Also, the changes that could appear prior to treatment are none or tend to reverse after the treatment is started.

The conducted examination showed that hypothyroidism does not significantly affect aberrations, neither corneal nor internal. The reason for differences obtained in the total eye horizontal coma aberrations and the corneal horizontal secondary coma could be the effect of hypothyroidism or other variables that are beyond the scope of this research. With a larger number of participants in both the examined and control groups, the research's sensitivity and specificity could be improved.

\section{CONCLUSION}

Overall, if hypothyroidism is treated correctly, it has an insignificant impact on the ocular surface and internal optics such as an intraocular crystalline lens and vitreous. The exception is the total eye horizontal coma, which could be slightly more 
positive in the eyes of patients with hypothyroidism, and corneal horizontal secondary coma, which could be slightly more negative in hypothyroidic patients. It leads to the conclusion that treated hypothyroidism has an insignificant impact when performing standard subjective eye exams. However, in wavefront-guided refractive surgery, the mean value of horizontal coma in the eyes of patients with treated hypothyroidism can vary from the mean values observed in patients without hypothyroidism.

This research does not show the eye condition in patients with non-treated hypothyroidism. To assess this, further evaluation should be carried out.

\section{Acknowledgements}

I would like to thank Dr. Joaquin Vidal and Mr. Raúl Pérez for MSc supervision as well as the Optotech Medical team for sharing the aberrometer for this research.

\section{Conflict of interests}

None.

\section{Funding}

This research did not receive any specific grant from funding agencies in the public, commercial, or not -for-profit sectors.

\section{REFERENCES}

1. Imam SK, Ahmad IS. Thyroid Disorders. Basic Science and Clinical Practise. Springer, Switzerland 2016: Springer.
2. Lin YY, Carrel H, Wang IJ, et al. Effect of Tear Film Break-up on Higher Order Aberrations of the Anterior Cornea in Normal, Dry, and PostLASIK Eyes. J Refract Surg. 2005; 21(5): 525-529, doi: 10.3928/1081. 597x-20050901-21, indexed in Pubmed: 16209455.

3. Dubbs SB, Spangler R. Hypothyroidism: causes, killers, and lifesaving treatments. Emerg Med Clin North Am. 2014; 32(2): 303-317, doi: 10.1016/j.emc.2013.12.003, indexed in Pubmed: 24766934.

4. Vaidya B, Pearce SHS. Management of hypothyroidism in adults. BMJ. 2008; 337: a801, doi: $10.1136 / \mathrm{bmj} . \mathrm{a} 801$, indexed in Pubmed: 18662921.

5. Persani L. Clinical review: Central hypothyroidism: pathogenic, diagnostic, and therapeutic challenges. J Clin Endocrinol Metab. 2012; 97(9): 3068-3078, doi: 10.1210/jc.2012-1616, indexed in Pubmed: 22851492.

6. Devdhar M, Ousman YH, Burman KD. Hypothyroidism. Endocrinol Metab Clin North Am. 2007; 36(3): 595-615, v, doi: 10.1016/j. ecl.2007.04.008, indexed in Pubmed: 17673121.

7. Unterhorst $H$, Rubin A. Ocular aberrations and wavefront aberrometry: A review. Afric Vis Eye Health. 2015; 74(1): a21, doi: 10.4102/aveh. v74i1.21.

8. Cerviño A, Hosking SL, Montes-Mico R, et al. Clinical Ocular Wavefront Analyzers. J Refract Surg. 2007; 23(6): 603-616, doi: 10.3928/1081597x-20070601-12, indexed in Pubmed: 17598581.

9. Oberholzer M, Gillan W, Rubin A. Higher order aberrations of the eye: Part one. Afric Vis Eye Health. 2016; 75(1), doi: 10.4102/aveh. v75i1.321.

10. Atchison DA, Suheimat M, Mathur A, et al. Anterior Corneal, Posterior Corneal, and Lenticular Contributions to Ocular Aberrations. Invest Ophthalmol Vis Sci. 2016; 57(13): 5263-5270, doi: 10.1167/iovs.1620067, indexed in Pubmed: 27701637.

11. Charman WN. Wavefront technology: past, present and future. Cont Lens Anterior Eye. 2005; 28(2): 75-92, doi: 10.1016/j. clae.2005.02.003, indexed in Pubmed: 16318838.

12. Philip K, Martinez A, Ho A, et al. Total ocular, anterior corneal and lenticular higher order aberrations in hyperopic, myopic and emmetropic eyes. Vision Res. 2012; 52(1): 31-37, doi: 10.1016/j. visres.2011.10.018, indexed in Pubmed: 22100815.

13. Gatzioufas Z, Panos G, Brugnolli E, et al. Corneal Topographical and Biomechanical Variations Associated With Hypothyroidism. J Refract Surg. 2014; 30(2): 78-79, doi: 10.3928/1081597x-20140120-01, indexed in Pubmed: 24477035. 